\title{
ON THE DETERMINATION OF THE RAMIFICATION INDEX IN CLIFFORD'S THEOREM
}

\author{
by ROBERT W. VAN DER WAALL
}

(Received 6th February 1987)

(Dedicated to Professor Dr B. Huppert on his sixtieth birthday)

\section{Introduction}

Let $K$ be a field, $G$ a finite group, $V$ a (right) $K G$-module. If $H$ is a subgroup of $G$, then, restricting the action of $G$ on $V$ to $H, V$ is also a $K H$-module. Notation: $V_{H}$.

Suppose $N$ is a normal subgroup of $G$. The $K N$-module $V_{N}$ is not irreducible in general, even when $V$ is irreducible as $K G$-module. A part of the well-known theorem of A. H. Clifford [1, V.17.3] yields the following.

Theorem (A. H. Clifford). Let $V$ be an irreducible $K G$-module. Let $N \leqslant G$. Then $V$ is a completely reducible $\mathrm{KN}$-module. Moreover

$$
V_{N}=V_{1}+\cdots+V_{n}
$$

where $V_{i} \not V_{j}$ as $K N$-modules if $i \neq j$, and each $V_{i}$ is a direct sum of $e$ isomorphic copies of an irreducible $K N$-submodule $W_{i}$, say, and $W_{i} \neq W_{j}$ if $i \neq j$. We write $V_{i}=e W_{i}$. It holds that

$$
V_{N} \simeq e\left(W_{1} \dot{+} \cdots \dot{+} W_{n}\right)=\underbrace{W_{1} \dot{+} \cdots \dot{+} W_{1}}_{\text {e times }} \dot{+\cdots}+\underbrace{W_{n} \dot{+} \cdots \dot{+} W_{n}}_{\text {e times }} .
$$

There exists a group $A$ with $N \subseteq A \subseteq G,|G: A|=n$, and a $K A$-submodule $T$ of $V$ such that $T_{N}=e W_{1}$ and $V \simeq T \bigotimes_{K A} K G$, as $K G$-modules. The integer $e$ is called the inertia index (or ramification index) of $V$ over $N$. It is independent of $i \in\{1, \ldots, n\}$.

Consider the groups $G, A, N$ as in Clifford's Theorem. It is important to know what the actual value of $e$ is, in particular whether $e$ divides the order of $A / N$. In two wellknown cases it is indeed true that $e$ divides $|A / N|$, namely

1. $K$ algebraically closed of characteristic zero or of positive characteristic not dividing the order of $G$ (see [6, p. 35]).

2. $K$ a finite field of odd characteristic not dividing the order of $G$ and containing.the primitive $m$ th-roots of unity, where $m=|G|_{2^{\prime}}, G / N$ an elementary abelian $p$-group (see [4, Theorem 13]), due to W. Willems. 
It is not true that the divisibility property of the inertia index always holds. As an example, take $R$ cyclic of order $3, K=\mathbb{F}_{2},\{1\}=N \triangleleft R$. Then there exists an irreducible two-dimensional $\mathbb{F}_{2}$-representation of $R$ with inertia index 2 over $N$. So here $e \nmid|R / N|$.

In [5, Theorem E], it was shown that $e=1, e=q$ or $e \mid q-1$ in the case where $G / N$ has prime order $q, G$ arbitrary finite, $K$ any finite field. It is the purpose of this paper to generalize that Theorem $\mathrm{E}$ of [5], and to give full information about the number $e$ in the case where $G / N$ is cyclic of prime power order and $K$ is a finite field. It follows from Clifford's Theorem that it is sufficient to consider the homogeneous case $V_{N}=e W, W$ an irreducible $K N$-submodule of the irreducible $K G$-module $V$. As a corollary to our results we conclude that $e$ always divides $|G / N|$ in the case where $G / N$ is a cyclic 2-group and $K$ is a finite field.

Most of the notation is standard and can be found in $[1,2,3]$ or is otherwise clear or self-explanatory. We use:

$\bar{E}=$ an algebraic closure of the field $E$,

$\mathbb{F}_{t}=$ finite field consisting of $t$ elements,

$\mathbb{F}(\chi)$ : see the definition given in the last lines of page 151 of [3].

This paper is dedicated to Professor Dr B. Huppert on the occasion of his sixtieth birthday, as a token of homage to him for all his work in finite group theory. Needless to say the books he has written will be landmarks for ever.

\section{The theorems and their proofs}

Theorem 1. Let $G$ be a finite group, $N \leqslant G, G / N$ cyclic of order $q^{n}, q$ prime. Assume $V$ is an irreducible $\mathbb{F} G$-module for a certain finite field $\mathbb{F}$. Suppose $V_{N}$ is a direct sum of $e$ irreducible $\mathbb{F} N$-submodules, each isomorphic to the irreducible $\mathbb{F} N$-submodule $U$ of $V_{N}$. We write $V_{N}=e U$. Let $\mathbb{F}(\eta)=\mathbb{F}(\eta(n) \mid n \in N)$, where $\eta$ is the trace function of some irreducible constituent of the $\overrightarrow{\mathbb{F}} N$-module $U \bigotimes_{\mathbb{F}} \overrightarrow{\mathbb{F}}$. Put $X \supseteq N,|G / X|=q$. Suppose $V_{X}$ is homogeneous but not irreducible. Then $e \geqq 2$ and either (1) or (2) holds.

1. Suppose $e=q^{a}, a \geqq 1$. Then (char $\left.\mathbb{F}, q\right)=1$ and $q|| \mathbb{F}(\eta) \mid-1$. There exists an irreducible $\mathbb{F} M$-submodule $W$ of $V$ such that $N \leqslant M \leqslant G,|G / M|=q^{a}, V_{M}=q^{a} W, W_{M}=U$, unless $q=2$ and $|\mathbb{F}(\eta)| \equiv-1(\bmod 4)$. If $q=2$ and $2^{\beta}|| \mathbb{F}(\eta)\left|+1,2^{\beta+1} \nmid\right| \mathbb{F}(\eta) \mid+1, \beta \geqq 2$, then there exists an irreducible $\mathbb{F} M$-submodule $W$ of $V$ with $N \leqslant M \leqslant G,|G / M|=2^{a+\beta-1}$, $V_{M}=2^{a} W, W_{N}=U$, unless $e=2$.

2. Suppose $e$ does not divide $|G / N|$. Then (char $\mathbb{F}, q)=1$. It holds that $e$ divides $\phi(|G / N|)=q^{n-1}(q-1)$. So $q$ is odd. Write $e=f q^{\gamma-1}, f \mid q-1$. Then $2 \leqq f$ and $f$ is the order of $|\mathbb{F}(\eta)|$ modulo $q$. When $\gamma \geqq 2$, then there exists an irreducible $\mathbb{F} M$-submodule $W$ of $V$ with $N \leqslant M \leqslant G,|G / M|=q^{a}, V_{M}=e W, W_{N}=U$, where $\left.q^{a}|| \mathbb{F}(\eta)\right|^{e}-1$ but $q^{a+1} \chi|F(\eta)|^{e}-1$.

It follows from the construction of the proof of Theorem 1, that each of the cases actually occurs in practice.

The following observation elucidates why the case $e=2$ deserves separate treatment.

Remark 2. Let $K$ be a finite field such that $2^{\beta}|| K\left|+1,2^{\beta+1} \chi\right| K \mid+1, \beta \geqq 2$. Let $G$ be 
a cyclic group of order $2^{n} \geqq 4$. Then $G$ admits a two-dimensional irreducible $K$ representation. Its restriction to $N=\{1\}$ is twice the trivial $K$-representation of $\{1\}$.

Proof of Theorem 1. It turns out that $q \neq$ char $\mathbb{F}$ for otherwise Green's Theorem VII.9.19 of [2] yields $e=1$. By Theorem VII.2.6 of [2] there exists a finite field $K$ containing $\mathbb{F}$ such that $K$ is a splitting field for $G$ and all its sections. Consider $V \bigotimes_{F} K$. Then, for suitable integers $u$ and $s$, we have the foilowing decompositions into irreducible $K G$-modules $R_{j}$ and irreducible $K N$-modules $T_{j}$ :

$$
V \bigotimes_{F} K=R_{1}+\cdots+R_{u}, \quad(e U) \bigotimes_{F} K \cong e\left(U \bigotimes_{F} K\right)=e\left(T_{1}+\cdots+T_{s}\right) .
$$

By [2, VII.1.16(e)] it follows that the $R_{i}$ are pairwise non-isomorphic absolutely irreducible $K G$-modules affording characters which are galois conjugated to each other; see also $[4,9.21]$. The same statement holds for the $K N$-modules $T_{i}$.

(1) Let $R_{1 \mid N}$ be not homogeneous. Let $W$ be an irreducible constituent of the $K N$ module $R_{1 \mid N}$. By considering the algebraic closure of $K$, the fact that $G / N$ is cyclic, Clifford's Theorem, the fact that char $K \Varangle|G / N|$, and combining these with Theorems 9.9. and 9.18 of Chapter VII of [2], it follows that $R_{1 \mid N}$ is a direct sum of pairwise non-isomorphic $K N$-submodules of $R_{1 \mid N}$. Such a $W$ is isomorphic to some $T_{i}$, as $K N$ modules. It follows that, say, $R_{1 \mid N} \cong T_{1}+\cdots+T_{f}$. The inertia group $I$ of $T_{1}$ in $G$ is here different from $G$ by assumption. Hence $X \supseteq I \supseteq N$ as $G / N$ is cyclic of prime power order. The ramification index of $R_{1}$ over $X$ is one (by [2, VII.9.18]) and $f=|G / I| \geqq 2$. Therefore there exists an irreducible $K I$-module $D$ with $D \bigotimes_{K I} K G \cong R_{1}$. Hence $D \bigotimes_{K I} K X$ is an irreducible constituent of $R_{1 \mid X}$. So $R_{1 \mid X}$ decomposes into a direct sum of $q$ pairwise non-isomorphic irreducible $K X$-modules, again by [2, VII.9.18]. Each of these modules gives $R_{1}$ when induced up to $G$. By galois conjugacy something similar holds for each of the $R_{1}, \ldots, R_{u}$. It follows that $\left(V\left(\chi_{F} K\right)_{X}\right.$ is a direct sum of pairwise non-isomorphic irreducible $K N$-modules. However this is in conflict with the assumption that $V_{X}$ should be homogeneous but not irreducible.

(2) Suppose now that all $R_{i \mid N}$ are homogeneous. Then, by [2, VII. 9.9] and [2, VII. 9.18] combined with the facts $G / N$ cyclic, Clifford's Theorem, char $K \Varangle|G / N|$, it now follows that all the $R_{i \mid N}$ are absolutely irreducible $K N$-modules. Let now $\chi$ be the trace function of $R_{i}$. Note that the field $F(\chi)$ does not depend on the index $i$, by [4, 9.21(c)]. So we have $V \bigotimes_{F} \mathbb{F}(\chi)=S_{1} \dot{+} \cdots \dot{+} S_{u}$, where, say, $R_{i} \cong S_{i} \bigotimes_{\mathrm{F}(x)} K$, and where $S_{i} ¥ S_{j}$ if $i \neq j$, as $\mathbb{F}(\chi) G$-modules. Observe that any $S_{i}$ is an absolutely irreducible $\mathbb{F}(\chi) G$-module. Since $R_{i \mid N}$ is an absolutely irreducible $K N$-module, it holds that $S_{i \mid N}$ is an absolutely irreducible $\mathbb{F}(\chi) N$-module, for any $i$. Notice that $u=[\mathbb{F}(\chi): \mathbb{F}]=$ $|\mathrm{Gal}(\mathbb{F}(\chi) / \mathbb{F})|$ and that $\mathrm{Gal}(\mathbb{F}(\chi) / \mathbb{F})$ is cyclic, generated by the Frobenius automorphism $x \mapsto x^{\mid F^{\mid}}$, any $x \in \mathbb{F}(\chi)$. We have $e t=u$, where $t$ is determined by $\left(V \bigotimes_{F} \mathbb{F}(\chi)\right)_{N}=$ $\left(S_{1} \dot{+} \cdots+S_{u}\right)_{N} \cong e\left(U \bigotimes_{F} \mathbb{F}(\chi)\right)=e\left(L_{1} \dot{+} \cdots+L_{t}\right) ;$ the $L_{i}$ are pairwise non-isomorphic (absolutely) irreducible $\mathbb{F}(\chi) N$-submodules of $U \bigotimes_{\mathbb{F}} \mathbb{F}(\chi)$. Consider $\mathbb{F}(\eta)$, where $\eta$ is the trace function of an irreducible $\bar{K} N$-submodule of $U \bigotimes_{\mathrm{F}} \bar{K}$. Hence $F(\eta) \subseteq K$. Then $t=s$, where $U \bigotimes_{F} \mathbb{F}(\eta)=Y_{1} \dot{+} \cdots+Y_{s}$ is the decomposition into (absolutely) irreducible $\mathbb{F}(\eta)$-submodules of $U \bigotimes_{F} \mathbb{F}(\eta)$. Since $\operatorname{Gal}(K / \mathbb{F})$ is cyclic, it is easily seen that $\mathbb{F}(\eta) \subseteq \mathbb{F}(\chi)$. 
(2.1) Assume from now on that $e \geqq 2$. Then some of the $S_{i \mid N}$ are isomorphic to one and the same (absolutely) irreducible $\mathbb{F}(\chi) N$-submodule $A$ of $U \bigotimes_{\mathrm{F}} \mathbb{F}(\chi)$. Let the cardinality of the set $S$, consisting of all these $S_{i}$ with $S_{i \mid N} \cong A$, be $c$. Without loss of generality, put $S=\left\{S_{1}, S_{2}, \ldots, S_{c}\right\}$. Then, just as it is done in [5, Theorem E, Proof], it follows that $c s=u=c t$ and that $c=\left|\operatorname{Gal}\left(\mathbb{F}(\chi) / \mathbb{F}_{r}\right)\right|$, where $r=|\mathbb{F}|^{s}$. Hence $c=e$. Let $\langle\gamma\rangle=\operatorname{Gal}\left(\mathbb{F}(\chi) / \mathbb{F}_{r}\right)$. Then, according to $[2$, VII.9.13], a unique one-dimensional $\mathbb{F}(\chi) G-$ module $\Lambda$ exists, on which $N$ acts trivially, such that $S_{1}^{\gamma} \cong S_{1} \bigotimes_{\mathrm{F}(x)} \Lambda$. By iteration and employing [2, VII.9.12(c)], it follows that $\Lambda^{n}$ is equal to the trivial one-dimensional $\mathbb{F}(\chi) G$-module, where $h=\left(r^{e}-1\right) /(r-1)$. The order of $\Lambda$ divides $|G / N|$. So $|\Lambda|=q^{a}, a \leqq n$. Since $1 \neq \gamma, a \geqq 1$ holds. Further, it follows from a repeated application of $\gamma$ that no integer $\left(r^{i}-1\right) /(r-1)$ is a multiple of $q^{a}$ for any $i=1, \ldots, e-1$. We now consider two possibilities: (1) $q \mid r-1$, (2) $q \nmid r-1$.

(2.1.1) Let $q \mid r-1$. Suppose $q^{m} \mid r-1$ but $q^{m+1} \chi r-1$. In the cases $\{q$ odd, $m \geqq 1\}$ and $\{q=2, m \geqq 2\}$ it follows that the integer $\left(r^{\alpha^{k} g}-1\right) /(r-1)$ with $q \nmid g$ and $k \geqq 0$, has precisely $k$ divisors $q$ in its prime decomposition. From this it follows that $e=q^{a}$. On the other hand, in the case $\{q=2, m=1\}$ we can write $r=2^{\beta} v-1$ with $\beta \geqq 2,2 \nmid v$. It follows that for $k \geqq 1,2 \nmid g$, the integer $\left(r^{2^{k}}-1\right) /(r-1)$ has precisely $\beta+k-1$ divisors 2 in its prime decomposition. Observe that for $k=0\left(r^{g}-1\right) /(r-1)$ is odd.

Suppose for the moment that $m \neq 1$. Then order $\Lambda=q^{a}=e(q=2$ is possible here). Let $N \subseteq M \subseteq G, M \leqslant G,|G / M|=q^{a}$. Then $\Lambda_{M}$ is the trivial one-dimensional $\mathbb{F}(\chi) M$ module. Hence $S_{1 \mid M} \cong S_{2 \mid M} \cong \cdots \cong S_{e \mid M}$, and $S_{1 \mid M}$ is an irreducible $\mathbb{F}(\chi) M$-module. Since $S_{1 \mid N}$ is an absolutely irreducible $\mathbb{F}(\chi) N$-module, we see that $S_{1 \mid M}$ is even absolutely irreducible as an $\mathbb{F}(\chi) M$-module. Consider $V_{M}=d\left(X_{1}+\cdots \dot{+} X_{z}\right), d \geqq 1$, the $X_{i}$ 's irreducible $\mathbb{F} M$-submodules of $V, X_{i} \neq X_{j}$ if $i \neq j$, as $\mathbb{F} M$-modules. Using $S_{1 \mid M} \cong S_{i \mid M}$ for each $i=1, \ldots, e$, it follows from the Deuring-Noether Theorem applied on the irreducible constituents of $V_{M} \bigotimes_{F} \mathbb{F}(\chi)$, that $e \leqq d$.. However, regarding $e U=V_{N}$ via $V_{M \mid N}, d \leqq e$ holds. Hence $d=e, z=1$ and so $V_{M}=e X_{1}=q^{a} X_{1}, X_{1 \mid N} \cong U$.

Now let $m=1, q=2, r=2^{\beta} v-1, \beta \geqq 2,2 \nmid v$. We see that if $e=2^{\delta}$ with $\delta \geqq 2$, then order $\Lambda=2^{\beta+\delta-1}$. Conversely, when order $\Lambda=2^{\beta+w}, w \geqq 1$, then $e=2^{w+1}$. Just as before it now follows that there exists an irreducible $\mathbb{F} \vec{M}$-submodule $W$ of $V$ with $N \leqslant M \leqslant G,|G / M|=2^{\beta+\delta-1}, V_{M}=2^{\delta} W, W_{N}=U$. The case $e=2$ is treated in Remark 2.

(2.1.2) Let $q \nmid r-1$. Since also $q \nmid r=|\mathbb{F}|^{s}$, it follows that $q$ is odd. Then obviously $e$ is equal to the order of $r$ modulo $q^{a}$. Hence $e \mid q^{a-1}(q-1)$, by Euler's Theorem. Hence, as $q^{a}|| G / N \mid$, we have $e \mid \phi(|G / N|)=q^{n-1}(q-1)$. So write $e=q^{\gamma-1} f, 1 \leqq \gamma \leqq a, f \mid q-1$. Observe $f \geqq 2$. For otherwise, just by $(r-1, q)=1=(r, q)$, we get a contradiction. Now suppose that $\gamma \geqq 2$ and that $q^{\varepsilon} \mid r^{\delta q^{\gamma-1}}-1, \varepsilon>a, q^{\varepsilon+1} \chi r^{\delta q^{\gamma-1}}-1$. Then $q^{\varepsilon-1} \mid r^{f q^{\gamma-2}}-1$, whence $q^{a} \mid r^{\delta q^{\gamma-2}}-1$, contrary to the definition of $e$. Hence we conclude that $q^{a} \mid r^{e}-1$, $q^{a+1} \nmid r^{e}-1$ if $\gamma \geqq 2$. Let $\hat{f}$ be the order of $r$ modulo $q$. Hence $f \mid f$. Also $q^{a} \mid r^{\delta q^{d-1}}-1$, whence $e \mid \hat{f} q^{a-1}$ by definition of $e$. Since $(q, f)=1, f q^{\gamma-1} \mid \hat{f} q^{a-1}$ implies $f \mid \hat{f}$. Therefore $\hat{f}=f$. Again by the same reasoning as in (2.1.1) the required result on the $\mathbb{F} M$-module $W$ now follows. Next suppose $\gamma=1$. Here $e=f$ is the order of $r$ modulo $q^{a}$. When $a \geqq 2$, then order $\hat{f}$ of $r$ modulo $q^{a-1}$ divides $f$. Also $q^{a} \mid \dot{r}^{\vec{j} q}-1$ and so $f \mid \hat{f} q$. Hence $f \mid \hat{f}$, whence $f=\hat{f}$. Inductively it follows that $f$ is the order of $r$ modulo $q$.

The proof of Theorem 1 is complete. 
Proof of Remark 2. Let $m=\min \{n, \beta+1\}$. Suppose $\zeta$ is a primitive $2^{m}$-th root of unity in some extension field of $K$. Hence $\zeta$ can be regarded as an element from $\mathbb{F}_{|K|^{2}} \supseteq K$, but here $\zeta \notin K$. It follows that the Frobenius automorphism $x \rightarrow x^{|K|}\left(x \in \mathbb{F}_{|K| 2}\right)$ of $\mathbb{F}_{|K|^{2}}$ has order 2 and $\zeta^{|K|} \neq \zeta$. By Exercise (9.6) of [4] there exists now an irreducible (possibly non-faithful) 2-dimensional $K$-representation of $G$.

We come now to the final Theorem 3 in which the decomposing behaviour of $V_{N}$ is demonstrated via normal subgroups between $G$ and $N$.

Theorem 3. Let $G / N$ be cyclic of order $q^{n}, q$ prime. Assume $V$ is an irreducible $\mathbb{F} G$ module for a finite field $\mathbb{F}$. Suppose $V_{N}=e U, U$ an irreducible $\mathbb{F} N$-submodule of $V_{N}$. Then precisely one of the following holds.

(1) If $N \leqslant M \leqslant G,|G / M|=q$, then $V_{M}$ is the direct sum of $q$ pairwise non-isomorphic irreducible $\mathbb{F} M$-submodules.

(2) There exists $N \leqslant T \leqslant G$ such that $V_{T}$ is an irreducible $\mathbb{F} T$-module and either $e=1$ or Theorem 1 holds for $\left\{T, V_{T}, N, U\right\}$.

In either case e divides $q^{n}(q-1)$ and $e \leqq q^{n}$.

Proof. We may assume that (1) does not hold and that $e \geqq 2$. Hence Theorem 1 holds for $\{G, V, N, U\}$ or, setting $N \leqslant M \leqslant G$ with $|G / M|=q, V_{M}$ is irreducible as an $\mathbb{F} M$ module. So we assume that $V_{M}$ is an irreducible $\mathbb{F} M$-module. Take any subgroup $S$ of $M$ with $N \subseteq S \subseteq M$. Note that $N \neq M$ by $e \geqq 2$. If $V_{S}$ is not homogeneous, then the property $G / S$ cyclic of prime power order would yield that $V_{M}$ would not be homogeneous, a contradiction. Hence $V_{S}$ is homogeneous. So there exists $N \subseteq L \subseteq T \subseteq M \equiv G$ such that $V_{T}$ is an irreducible $\mathbb{F} T$-module and such that $V_{L}$ is homogeneous but not irreducible as an $\mathbb{F} L$-module, where $|T / L|=q$. Therefore Theorem 1 holds for $\left\{T, V_{T}, N, U\right\}$.

In case (1) we put $V_{M}=L_{1} \dot{+} \cdots+L_{q}$ as the required sum decomposition. Hence $L_{i \mid N}=e q^{-1} U$ for each $i$, by the Krull-Schmidt Theorem. So by induction $e \mid q^{n}(q-1)$ and $e \leqq q^{n}$.

If in case (2) $T \neq G$ then by induction $e \| T / N \mid(q-1)$ and $e \leqq|T / N|$. Hence $e \mid q^{n}(q-1)$ and $e \leqq q^{n}$.

If in case (2) $T=G$ then we read of directly from Theorem 1 that $e \mid q^{n}(q-1)$ and $e \leqq q^{n}$.

\section{REFERENCES}

1. B. HuPPERT, Endliche Gruppen I (Springer-Verlag, Berlin-Heidelberg-New York, 1967).

2. B. Huppert and N. Blackburn, Finite Groups II (Springer-Verlag, Berlin-Heidelberg-New York, 1982).

3. I. M. IsaAcs, Character Theory of Finite Groups (Academic Press, New York-London, 1976).

4. R. W. van der WaAlL, Minimal non-M-groups, Indag. Math. 42 (1980), 93-106.

5. R. W. VAN DER WaAl, On Clifford's theorem and ramification indices for symplectic modules over a finite field, Proc. Edinburgh Math. Soc. 30 (1987), 153-167. 
6. W. WILLEMS, Induzierte und eingeschränkte Moduln über Gruppenringen (Diplomarbeit, Mainz, 1973).

Mathematisch Instituut

UNIVERSITEIT VAN AMSTERDAM

ROETERSSTRAAT 15

1018 WB AMSTERDAM

The Netherlands 